DESY-01-085

July 2001

PITHA 01/06

TTP01-16

\title{
Top Quark Spin Correlations at Hadron Colliders: Predictions at Next-to-Leading Order QCD
}

\author{
W. Bernreuther ${ }^{a}$, A. Brandenburg ${ }^{b}$,, Z. G. Si ${ }^{a}$ and P. Uwer ${ }^{c}$ \\ ${ }^{a}$ Institut für Theoretische Physik, RWTH Aachen, 52056 Aachen, Germany \\ ${ }^{b}$ DESY-Theorie, 22603 Hamburg, Germany \\ ${ }^{c}$ Institut für Theoretische Teilchenphysik, Universität Karlsruhe, 76128 Karlsruhe, \\ Germany
}

\begin{abstract}
:
The collider experiments at the Tevatron and the LHC will allow for detailed investigations of the properties of the top quark. This requires precise predictions of the hadronic production of $t \bar{t}$ pairs and of their subsequent decays. In this Letter we present for the reactions $p \bar{p}, p p \rightarrow t \bar{t}+X \rightarrow \ell^{+} \ell^{\prime-}+X$ the first calculation of the dilepton angular distribution at next-to-leading order (NLO) in the QCD coupling, keeping the full dependence on the spins of the intermediate $t \bar{t}$ state. The angular distribution reflects the degree of correlation of the $t$ and $\bar{t}$ spins which we determine for different choices of $t$ and $\bar{t}$ spin bases. In the case of the Tevatron, the QCD corrections are sizeable, and the distribution is quite sensitive to the parton content of the proton.
\end{abstract}

PACS numbers: 12.38.Bx, 13.88.+e, 14.65.Ha

\footnotetext{
* supported by BMBF, contract 05 HT1 PAA 4

${ }^{\dagger}$ supported by a Heisenberg fellowship of D.F.G.
} 
The top quark is by far the heaviest fundamental fermion discovered [1] to date. It is an excellent probe of the fundamental interactions in the high energy regime that will be explored by the upgraded Fermilab Tevatron collider and by the CERN large hadron collider LHC. It is expected that very large numbers of top quarks will be produced with these colliders: eventually about $10^{4}$ top quark-antiquark $(t \bar{t})$ pairs per year at the Tevatron and more than about $10^{7} t \bar{t}$ pairs per year at the LHC. This will make feasible precise investigations of the interactions of top quarks.

Because of their extremely short lifetime top quarks find no time to form hadronic bound states: they are highly instable particles whose interactions are governed by shortdistance dynamics [2]. As a consequence the properties of the top quark and antiquark, in particular phenomena associated with their spins, are reflected directly in the distributions and the corresponding angular correlations of the jets, $W$ bosons, or leptons into which the $t$ and $\bar{t}$ decay. These distributions are determined by the $t$ and $\bar{t}$ polarizations and spin correlations induced by the production mechanism(s). Furthermore they depend on the interactions responsible for the top (anti-)quark decay. Hence the analysis of these distributions will be an important tool, once large data samples will be available, to obtain detailed information about top-quark production and decay.

For hadronic pair production the spin correlations of $t \bar{t}$ pairs were studied to leading order in the coupling $\alpha_{s}$ of Quantum Chromodynamics (QCD) in ref. [3, 4]. In particular it was analyzed which spin bases are most suitable for the investigation of $t \bar{t}$ spin correlations induced by the strong interactions ${ }^{n}$. There exists also an extensive literature, for example [6] and references therein, on how to exploit top-quark spin phenomena at hadron colliders in the search for new interactions. The work which we report in this Letter serves the purpose of putting predictions of $t \bar{t}$ spin correlations within the standard model of particle physics (SM) on firmer grounds. We analyze the hadronic production of $t \bar{t}$ pairs and their subsequent decays, keeping the full information on the spin configuration of the $t \bar{t}$ state. We extend the existing results by taking into account the next-to-leading order (NLO) QCD corrections in the production and the decay of the $t \bar{t}$ pairs. More specifically we consider the channels where both $t$ and $\bar{t}$ decay semileptonically,

$$
p \bar{p}, p p \rightarrow t \bar{t}+X \rightarrow \ell^{+} \ell^{\prime-}+X,
$$

$(\ell=e, \mu, \tau)$, and we predict the following double leptonic distribution at NLO in the coupling $\alpha_{s}$ :

$$
\frac{1}{\sigma} \frac{d^{2} \sigma}{d \cos \theta_{+} d \cos \theta_{-}}=\frac{1}{4}\left(1+\mathrm{B}_{1} \cos \theta_{+}+\mathrm{B}_{2} \cos \theta_{-}-\mathrm{C} \cos \theta_{+} \cos \theta_{-}\right),
$$

with $\sigma$ being the cross section for the channel under consideration. In Eq. (2) $\theta_{+}\left(\theta_{-}\right)$ denotes the angle between the direction of flight of the lepton $\ell^{+}\left(\ell^{\prime-}\right)$ in the $t(\bar{t})$ rest frame $\hat{f}$ and a reference direction $\hat{\mathbf{a}}(\hat{\mathbf{b}})$. The directions $\hat{\mathbf{a}}, \hat{\mathbf{b}}$ can be chosen arbitrarily.

\footnotetext{
${ }^{1} \mathrm{~A}$ first attempt to measure $t \bar{t}$ spin correlations with a very small data sample was made in ref. [5].

${ }^{2}$ We define the rest frame of the $t(\bar{t})$ quark by a rotation-free Lorentz boost from the center-of-mass (c.m.) frame of the initial partons that produce the $t \bar{t}$ pair. If one defines the $t(\bar{t})$ rest frame by a boost from the hadronic c.m. frame, it will differ from our choice by a Wigner rotation.
} 
Different choices will yield different values for the coefficients $\mathrm{B}_{1,2}$ and $\mathrm{C}$. The physical interpretation of these coefficients is well known [3, \#]: The coefficient $\mathrm{C}$ in Eq. (2) reflects spin correlations of the $t \bar{t}$ intermediate state. A more detailed discussion will be given below Eq. (6). For our choices of the directions $\hat{\mathbf{a}}$ and $\hat{\mathbf{b}}$ (cf. Eq. (8)) QCD interactions yield vanishing coefficients $\mathrm{B}_{1}, \mathrm{~B}_{2}$.

In principle one could measure the angular distribution of every possible decay product of the top (anti-)quark. In the SM, where the main top-quark decay modes are $t \rightarrow b W \rightarrow b q \bar{q}^{\prime}, b \ell v_{\ell}$, the most powerful analyzers of the polarization of the top quark are the charged leptons, or the jets that originate from quarks of weak isospin $-1 / 2$ produced by the decay of the $W$ boson. Here we restrict ourselves to the double leptonic distribution.

To predict the "dilepton + jets" distribution (2) at NLO accuracy we have to consider the following parton subprocesses:

$$
\begin{gathered}
g g, q \bar{q} \stackrel{t \bar{t}}{\longrightarrow} b \bar{b} \ell^{+} \ell^{\prime-} \vee_{\ell} \overline{\mathrm{v}}_{\ell^{\prime}}, \\
g g, q \bar{q} \stackrel{t \bar{t}}{\longrightarrow} b \bar{b} \ell^{+} \ell^{\prime-} \mathrm{v}_{\ell} \overline{\mathrm{v}}_{\ell^{\prime}}+g, \\
g+q(\bar{q}) \stackrel{t \bar{t}}{\longrightarrow} b \bar{b} \ell^{+} \ell^{\prime-} \mathrm{v}_{\ell} \overline{\mathrm{v}}_{\ell^{\prime}}+q(\bar{q}) .
\end{gathered}
$$

At the Tevatron the cross section is dominated by quark-antiquark annihilation while at the LHC gluon-gluon fusion is predicted to be the dominant production process.

In view of the fact that the total width $\Gamma_{t}$ of the top quark is much smaller than its mass $m_{t}\left(\Gamma_{t} / m_{t}=O(1 \%)\right)$, one may analyze the above reactions using the so-called leading pole approximation [9]. This amounts to expanding the amplitudes of Eqs. (3) - (5) around the poles of the unstable $t$ and $\bar{t}$ quarks. Only the leading term of this expansion, i.e., the residue of the double poles is kept here. The radiative corrections to the respective lowest-order amplitudes can be classified into so-called factorizable and non-factorizable corrections. We take into account the factorizable corrections to the above reactions for which the squared matrix element $\mathcal{M}$ is of the form $|\mathcal{M}|^{2} \propto \operatorname{Tr}[\rho R \bar{\rho}]$. Here $R$ denotes the respective spin density matrix for the production of on-shell $t \bar{t}$ pairs, and $\rho(\bar{\rho})$ is the $t(\bar{t})$ decay density matrix.

To obtain a theoretical prediction for the distribution in Eq. (2) at NLO accuracy we use our recent results [7] on the $t \bar{t}$ production spin-density matrices at NLO QCD. These results extend previous calculations [8] of the differential $t \bar{t}$ cross section with spins summed over and allow the calculation of the cross section for a specific spin configuration of the $t \bar{t}$ state. In particular, the quantization axes can be chosen arbitrarily.

The decay density matrix $\rho(\bar{\rho})$ required for computing (2) describes the normalized angular distribution of the decay of a polarized $t(\bar{t})$ quark into $\ell^{+}\left(\ell^{-}\right)+$anything in the rest frame of the $t(\bar{t})$ quark. The matrix $\rho$ has the form $2 \rho_{\alpha^{\prime} \alpha}=\left(11+\kappa_{+} \sigma \cdot \hat{\mathbf{q}}_{+}\right)_{\alpha^{\prime} \alpha}$ where $\hat{\mathbf{q}}_{+}$describes the direction of flight of $\ell^{+}$in the rest frame of the $t$ quark and $\sigma_{i}$ denote the Pauli matrices. The decay matrix $\bar{\rho}$ is obtained from $\rho$ by replacing $\hat{\mathbf{q}}_{+}$by $-\hat{\mathbf{q}}_{-}$and $\kappa_{+}$

\footnotetext{
${ }^{3}$ This is due to the parity invariance of QCD.
} 
by $\kappa_{-}$. The factor $\kappa_{+}\left(\kappa_{-}\right)$signifies the top-spin analyzing power of the charged lepton. It is equal to one to lowest order in the SM, that is, for $V-A$ charged currents. Its value including the order $\alpha_{s}$ corrections can be extracted from the results of [10] and turns out to be very close to one: $\kappa_{+}=\kappa_{-}=1-0.015 \alpha_{s}$. Using the general expressions for $\rho$, $\bar{\rho}$ and the fact that the factorizable contributions are of the form $\operatorname{Tr}[\rho R \bar{\rho}]$ one obtains the following formula for the correlation coefficient $\mathrm{C}$ in Eq. (2):

$$
\mathrm{C}=4 \kappa_{+} \kappa_{-}\left\langle\left(\hat{\mathbf{a}} \cdot \mathbf{s}_{t}\right)\left(\hat{\mathbf{b}} \cdot \mathbf{s}_{\bar{t}}\right)\right\rangle,
$$

where $\mathbf{s}_{t}, \mathbf{s}_{\bar{t}}$ denote the $t$ and $\bar{t}$ spin operators. The expectation value in Eq. (6) is defined with respect to the matrix elements for the hadronic production of $t \bar{t} X$. It is related to the more familiar double spin asymmetries

$$
4\left\langle\left(\hat{\mathbf{a}} \cdot \mathbf{s}_{t}\right)\left(\hat{\mathbf{b}} \cdot \mathbf{s}_{\bar{t}}\right)\right\rangle=\frac{\mathrm{N}(\uparrow \uparrow)+\mathrm{N}(\downarrow \downarrow)-\mathrm{N}(\uparrow \downarrow)-\mathrm{N}(\downarrow \uparrow)}{\mathrm{N}(\uparrow \uparrow)+\mathrm{N}(\downarrow \downarrow)+\mathrm{N}(\uparrow \downarrow)+\mathrm{N}(\downarrow \uparrow)},
$$

where $N(\uparrow \uparrow)$ etc. denote the number of $t \bar{t}$ pairs with $t$ and $\bar{t}$ spin parallel - or anti-parallel - to $\hat{\mathbf{a}}$ and $\hat{\mathbf{b}}$, respectively. From Eq. (7) one can see that the axes $\hat{\mathbf{a}}, \hat{\mathbf{b}}$ introduced through the angles $\theta_{ \pm}$in Eq. (2) can be interpreted as quantization axes of the intermediate $t \bar{t}$ state within our approximation. Eq. (6) generalizes the lowest-order results of [3, 4] and holds for factorizable contributions to all orders in the QCD couplingf.

For definiteness we consider here the following spin bases:

$$
\begin{array}{lll}
\hat{\mathbf{a}}=\hat{\mathbf{k}}_{t}, & \hat{\mathbf{b}}=\hat{\mathbf{k}}_{\bar{t}} & \text { (helicity basis) } \\
\hat{\mathbf{a}}=\hat{\mathbf{p}}, & \hat{\mathbf{b}}=\hat{\mathbf{p}}, & \text { (beam basis) }, \\
\hat{\mathbf{a}}=\hat{\mathbf{d}}_{t}, & \hat{\mathbf{b}}=\hat{\mathbf{d}}_{\bar{t}} & \text { (off-diagonal basis) } .
\end{array}
$$

Here $\hat{\mathbf{k}}_{t}\left(\hat{\mathbf{k}}_{\bar{t}}\right)$ denotes the direction of flight of the $t(\bar{t})$ quark in the parton c.m.s., and $\hat{\mathbf{p}}$ is the unit vector along one of the hadronic beams in the laboratory frame. Furthermore $\hat{\mathbf{d}}_{t}$ is the axis constructed in ref. [4] with respect to which the spins of $t$ and $\bar{t}$ produced by $q \bar{q}$

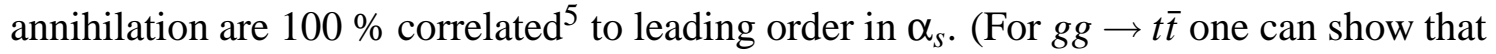
no spin basis with this property exists.)

Table 11 contains our results for $\mathrm{C}$ at leading and next-to-leading order in $\alpha_{s}$ using the parton distribution functions (PDF) CTEQ5L (LO) and CTEQ5M (NLO) of [12]. (These numbers and the results given below were obtained by integrating over the full phase phase. Results with cuts included will be given elsewhere [13].) For $p \bar{p}$ collisions at $\sqrt{s}=2 \mathrm{TeV}$ the helicity basis is not the best choice because the $t, \bar{t}$ quarks are only moderately relativistic in this case. Table 1 shows that the dilepton spin correlations at the Tevatron are large both in the off-diagonal and in the beam basis. In fact they are

\footnotetext{
${ }^{4}$ The non-factorizable NLO QCD corrections were calculated for $g g$ and $q \bar{q}$ initial states in ref. [11]. We expect with these results that the effect of these corrections on the dileptonic angular correlations is small.

${ }^{5}$ We use the definitions for $\hat{\mathbf{d}}_{t}$ and $\hat{\mathbf{d}}_{\bar{t}}$ given in ref. [7]. In particular, $\hat{\mathbf{d}}_{\bar{t}}=\hat{\mathbf{d}}_{t}$ at LO. The sign of $C_{\text {off. at }}$ LO is therefore opposite to that of [ $€$ ].

${ }^{6} \mathrm{We}$ use the $\overline{\mathrm{MS}}$ factorization scheme, $\alpha_{s}$ is defined to be the five-flavour $\overline{\mathrm{MS}}$ coupling, and $m_{t}$ is defined in the on-shell scheme.
} 
almost identical. The QCD corrections decrease the LO results for these correlations by about $10 \%$. Since the $g g$ initial state dominates $t \bar{t}$ production with $p p$ collisions at $\sqrt{s}=$ $14 \mathrm{TeV}$ the beam and off-diagonal bases are no longer useful. Here the helicity basis is a good choice and gives a spin correlation of about 30\%. In this case the QCD corrections are small. The large difference between the LO and NLO results for the correlation in the beam basis at the LHC is due to an almost complete cancellation of the contributions from the $q \bar{q}$ and $g g$ initial state at LO.

\begin{tabular}{|c|rr|rr|}
\hline & \multicolumn{2}{|c|}{$p \bar{p}$ at $\sqrt{s}=2 \mathrm{TeV}$} & \multicolumn{2}{c|}{$p p$ at $\sqrt{s}=14 \mathrm{TeV}$} \\
\cline { 2 - 5 } & \multicolumn{1}{|c}{ LO } & NLO & LO & NLO \\
\hline \hline $\mathrm{C}_{\text {hel. }}$ & -0.456 & -0.389 & 0.305 & 0.311 \\
$\mathrm{C}_{\text {beam }}$ & 0.910 & 0.806 & -0.005 & -0.072 \\
$\mathrm{C}_{\text {off. }}$ & 0.918 & 0.813 & -0.027 & -0.089 \\
\hline
\end{tabular}

Table 1: Coefficient $\mathrm{C}$ of Eq. (6) to leading (LO) and next-to-leading order (NLO) in $\alpha_{s}$ for the spin bases of Eq. (8). The parton distribution functions of [12] were used choosing the renormalization scale $\mu_{R}$ equal to the factorization scale $\mu_{F}=m_{t}=175 \mathrm{GeV}$.

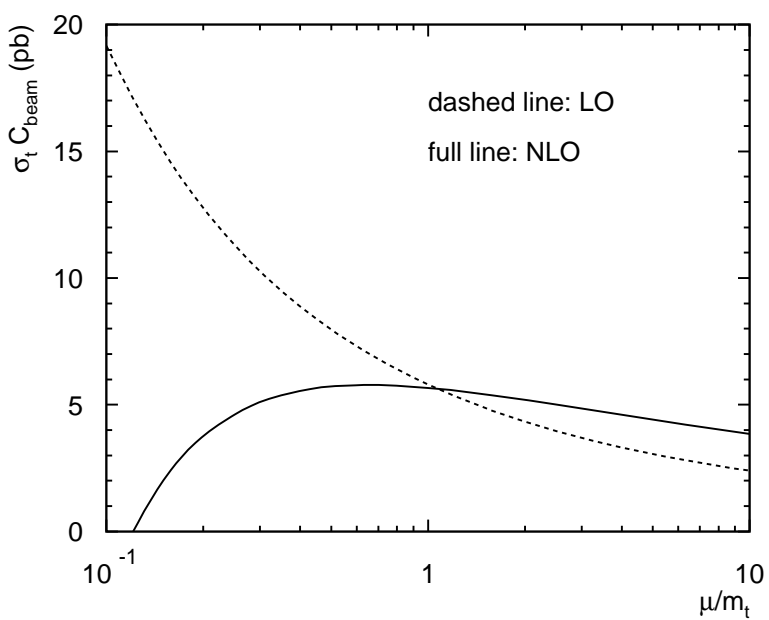

Figure 1: Dependence of $\sigma_{t} \mathrm{C}_{\text {beam }}$ at LO (dashed line) and at NLO (solid line) on $\mu=$ $\mu_{R}=\mu_{F}$ for p $\bar{p}$ collisions at $\sqrt{s}=2 \mathrm{TeV}$, with PDF of [12]].

We now discuss the uncertainties of our predictions. It is well known that the inclusion of the QCD corrections reduces the dependence of the $t \bar{t}$ cross section $\sigma_{t}$ on the renormalization and factorization scales significantly. The same is true for the product $\sigma_{t} \mathrm{C}$. 


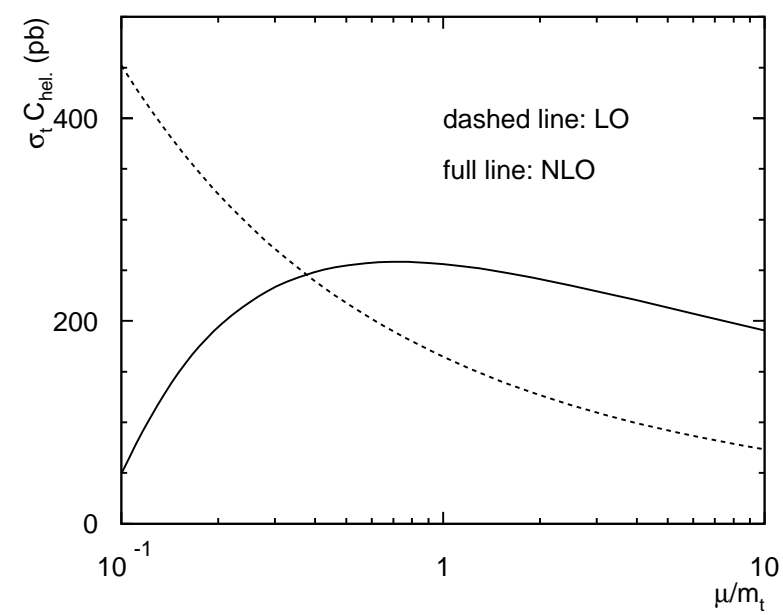

Figure 2: Dependence of $\sigma_{t} \mathrm{C}_{\text {hel. }}$ at $L O$ (dashed line) and at $N L O$ (solid line) on $\mu=\mu_{R}=$ $\mu_{F}$ for pp collisions at $\sqrt{s}=14 \mathrm{TeV}$, with PDF of [[2]].

In Figs. 11 and 2 we demonstrate this with $\sigma_{t} \mathrm{C}_{\text {beam }}$ and $\sigma_{t} \mathrm{C}_{\text {hel. }}$. evaluated at Tevatron and LHC energies, respectively, as functions of $\mu / m_{t}$, where $\mu=\mu_{R}=\mu_{F}$. The corresponding figure for $\sigma_{t} \mathrm{C}_{\text {off. }}$ is almost identical to Fig. 1.

To leading order in $\alpha_{s}$ the coefficient $\mathrm{C}$ depends only on the factorization scale $\mu_{F}$, while at NLO it depends on both scales $\mu_{R}$ and $\mu_{F}$. Table 2 shows our NLO results for the three choices $\mu_{R}=\mu_{F}=m_{t} / 2, m_{t}, 2 m_{t}$, again using the PDF of [12]. An extension of this work, which is however beyond the scope of this Letter, would be the resummation of Sudakov-type logarithms at the next-to-leading logarithmic level. This was performed in ref. [16] for the total cross section $\sigma_{t}$ and it stabilizes the predictions for $\sigma_{t}$ with respect to variations of $\mu_{R}$ and $\mu_{F}$.

\begin{tabular}{|c|ccc|c|}
\hline \multirow{2}{*}{$\mu_{R}=\mu_{F}$} & \multicolumn{3}{|c|}{$p \bar{p}$ at $\sqrt{s}=2 \mathrm{TeV}$} & $p p$ at $\sqrt{s}=14 \mathrm{TeV}$ \\
\cline { 2 - 5 } & $\mathrm{C}_{\text {hel. }}$ & $\mathrm{C}_{\text {beam }}$ & $\mathrm{C}_{\text {off. }}$ & $\mathrm{C}_{\text {hel. }}$ \\
\hline \hline$m_{t} / 2$ & -0.364 & 0.774 & 0.779 & 0.278 \\
$m_{t}$ & -0.389 & 0.806 & 0.813 & 0.311 \\
$2 m_{t}$ & -0.407 & 0.829 & 0.836 & 0.331 \\
\hline
\end{tabular}

Table 2: Dependence of the correlation coefficients, computed with the PDF of [12], on $\mu=\mu_{R}=\mu_{F}$ at $N L O$. 
In Table 3 we compare results for $\mathrm{C}$ using different sets of PDF. In the case of $p \bar{p}$ collisions at $\sqrt{s}=2 \mathrm{TeV}$, the spread of the results is larger than the scale uncertainty given in Table 2. To a considerable extent this is due to an interesting feature of $\mathrm{C}$, namely the $q \bar{q}$ and $g g$ initial states contribute to $\mathrm{C}$ with opposite signs. Therefore the spin correlations are quite sensitive to the relative weights of $q \bar{q}$ and $g g$ initiated $t \bar{t}$ events. These weights depend in particular on the chosen set of PDF. For example, one finds the following individual NLO contributions for the helicity, beam, and off-diagonal correlation at the upgraded Tevatron: for the GRV98 (MRST98) PDF $\mathrm{C}_{\mathrm{hel}}^{q \bar{q}}=-0.443(-0.486)$, $\mathrm{C}_{\text {hel. }}^{g g}=+0.124(+0.075), \mathrm{C}_{\text {beam }}^{q \bar{q}}=+0.802(+0.879), \mathrm{C}_{\text {beam }}^{g g}=-0.068(-0.042)$, and $\mathrm{C}_{\text {off. }}^{q \bar{q}}=+0.810(+0.889), \mathrm{C}_{\mathrm{off}}^{g g}=-0.073(-0.044)$. This suggests that accurate measurements of the dilepton distribution (2), using different spin bases, at the upgraded Tevatron may provide additional constraints in the continuing effort to improve the knowledge of the PDF.

\begin{tabular}{|c|ccc|c|}
\hline \multirow{2}{*}{ PDF } & \multicolumn{3}{|c|}{$p \bar{p}$ at $\sqrt{s}=2 \mathrm{TeV}$} & $p p$ at $\sqrt{s}=14 \mathrm{TeV}$ \\
\cline { 2 - 5 } & $\mathrm{C}_{\text {hel. }}$ & $\mathrm{C}_{\text {beam }}$ & $\mathrm{C}_{\text {off. }}$ & $\mathrm{C}_{\text {hel. }}$ \\
\hline \hline GRV98 & -0.325 & 0.734 & 0.739 & 0.332 \\
CTEQ5 & -0.389 & 0.806 & 0.813 & 0.311 \\
MRST98 & -0.417 & 0.838 & 0.846 & 0.315 \\
\hline
\end{tabular}

Table 3: Correlation coefficients $\mathrm{C}_{\text {hel. }}, \mathrm{C}_{\mathrm{beam}}$, and $\mathrm{C}_{\mathrm{off}}$ at $N L O$ for $\mu_{R}=\mu_{F}=m_{t}$ and different sets of parton distribution functions: GRV98 [14], CTEQ5 [12], and MRST98 $(c-g)[15]$.

Finally we have studied the dependence of the $\mathrm{C}$ coefficients on the top quark mass. For this we have used again the CTEQ5 PDF and set $\mu=m_{t}$. In the case of $p \bar{p}$ collisions at $\sqrt{s}=2 \mathrm{TeV}$, a variation of $m_{t}$ from 170 to $180 \mathrm{GeV}$ changes $\mathrm{C}_{\text {hel. }}$ from -0.378 to

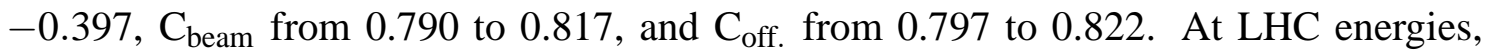
$\mathrm{C}_{\text {hel. }}$. changes by less than a percent.

The extension of our results to the "lepton+jets" and "all jets" decay channels [13] is straightforward. The "lepton+jets" channels should be particularly useful for detecting $t \bar{t}$ spin correlations: although one looses top-spin analyzing power one gains in statistics and the experimental reconstruction of the $t$ and $\bar{t}$ rest frames may also be facilitated.

In conclusion we have analyzed, at next-to-leading order in $\alpha_{s}$, the hadronic production of $t \bar{t}$ quarks in a general spin configuration and have computed the dileptonic angular correlation coefficients $\mathrm{C}$ that reflect the degree of correlation between the $t$ and $\bar{t}$ spins. Our results for the Tevatron show that the scale and in particular the PDF uncertainties in the prediction of the dileptonic angular distribution must be reduced before $t \bar{t}$ spin correlations can be used in a meaningful way to search for relatively small effects of new interactions that are, for example, not distinguished by violating parity or $\mathrm{CP}$ invariance. 
Our results may also be useful to learn more about the parton distributions in the proton at high energies. For $p p$ collisions at $\sqrt{s}=14 \mathrm{TeV}$ the theoretical uncertainties in the prediction of this distribution are smaller and one may adopt the optimistic view that at the time the LHC will be turned on further theoretical progress will have turned top quark spin correlations into a precision tool for the analysis of $t \bar{t}$ events.

\section{Acknowledgments}

We would like to thank S. Dittmaier, S. Moch and B. Plümper for useful discussions.

\section{References}

[1] F. Abe et al. [CDF Collaboration], Phys. Rev. Lett. 74, 2626 (1995); S. Abachi et al. [D0 Collaboration], Phys. Rev. Lett. 74, 2632 (1995).

[2] I. I. Bigi et al., Phys. Lett. B 181, 157 (1986).

[3] T. Stelzer and S. Willenbrock, Phys. Lett. B 374, 169 (1996); G. Mahlon and S. Parke, Phys. Rev. D 53, 4886 (1996); A. Brandenburg, Phys. Lett. B 388, 626 (1996); D. Chang, S.C. Lee and A. Soumarokov, Phys. Rev. Lett. 77, 1218 (1996).

[4] G. Mahlon and S. Parke, Phys. Lett. B 411, 173 (1997).

[5] B. Abbott et al. [D0 Collaboration], Phys. Rev. Lett. 85, 256 (2000).

[6] D. Atwood, A. Aeppli and A. Soni, Phys. Rev. Lett. 69, 2754 (1992); G. L. Kane, G. A. Ladinsky and C. P. Yuan, Phys. Rev. D 45, 124 (1992); C. R. Schmidt and M. E. Peskin, Phys. Rev. Lett. 69, 410 (1992); W. Bernreuther and A. Brandenburg, Phys. Lett. B 314, 104 (1993); W. Bernreuther, A. Brandenburg and M. Flesch, hepph/9812387; A. Brandenburg and J. P. Ma, Phys. Lett. B 298, 211 (1993); P. Haberl, O. Nachtmann and A. Wilch, Phys. Rev. D 53, 4875 (1996); K. Cheung, Phys. Rev. D 55, 4430 (1997); B. Grzadkowski, B. Lampe and K. J. Abraham, Phys. Lett. B 415, 193 (1997); W. Bernreuther, M. Flesch and P. Haberl, Phys. Rev. D 58, 114031 (1998); D. Atwood, S. Bar-Shalom, G. Eilam and A. Soni, Phys. Rept. 347, 1 (2001).

[7] W. Bernreuther, A. Brandenburg and Z. G. Si, Phys. Lett. B 483, 99 (2000); W. Bernreuther, A. Brandenburg, Z. G. Si and P. Uwer, Phys. Lett. B 509, 53 (2001).

[8] P. Nason, S. Dawson and R. K. Ellis, Nucl. Phys. B 303, 607 (1988); Nucl. Phys. B 327, 49 (1989); W. Beenakker, H. Kuijf, W. L. van Neerven and J. Smith, Phys. Rev. D 40, 54 (1989); W. Beenakker et al., Nucl. Phys. B 351, 507 (1991).

[9] R. G. Stuart, Phys. Lett. B 262, 113 (1991); A. Aeppli, G. J. van Oldenborgh and D. Wyler, Nucl. Phys. B 428, 126 (1994).

[10] M. Jezabek and J. H. Kühn, Nucl. Phys. B 320, 20 (1989); A. Czarnecki, M. Jezabek and J. H. Kühn, Nucl. Phys. B 351, 70 (1991). 
[11] W. Beenakker, F. A. Berends and A. P. Chapovsky, Phys. Lett. B 454, 129 (1999).

[12] H.L. Lai et al. [CTEQ Collaboration], Eur. Phys. J. C 12, 375 (2000).

[13] W. Bernreuther, A. Brandenburg, Z. G. Si and P. Uwer, in preparation.

[14] M. Glück, E. Reya and A. Vogt, Eur. Phys. J. C 5, 461 (1998).

[15] A.D. Martin, R.G. Roberts, W.J. Stirling, R.S. Thorne, Eur. Phys. J. C 4, 463 (1998).

[16] N. Kidonakis and G. Sterman, Nucl. Phys. B 505, 321 (1997); R. Bonciani, S. Catani, M. L. Mangano and P. Nason, Nucl. Phys. B 529, 424 (1998); N. Kidonakis, E. Laenen, S. Moch and R. Vogt, hep-ph/0105041. 